\title{
General dental practitioner views on providing alcohol related health advice; an exploratory study
}
IN BRIEF
- Alcohol constitutes a major global problem accounting for significant levels of worldwide morbidity and mortality.
- Alcohol is a widely recognised and accepted aetiological risk factor in the development of oral cancer.
- The universal and opportunistic delivery of alcohol related advice from all health professionals has the potential to positively impact on alcohol related harm.

\author{
S. Shepherd, ${ }^{1}$ L. Young, ${ }^{2}$ J. E. Clarkson, ${ }^{3}$ D. Bonetti ${ }^{4}$ and G. R. Ogden ${ }^{5}$
}

Objective To identify salient beliefs of general dental practitioners (GDPs) regarding their role in the identification of alcohol misuse and the provision of an alcohol related health message in the primary dental care setting. Method A convenience sample of 12 GDPs practising in the North Highland region of Scotland underwent semi-structured interview. An inductive approach was used with subsequent basic thematic content analysis performed on the transcripts. Results GDPs universally agreed that alcohol consumption plays a role in both oral health and general health but this did not translate into effective communication about alcohol during dental consultation. Current knowledge of recommended safe alcohol consumption guidelines was poor - evidence of potential GDP training requirements. The primary barriers related to disruption of the clinician-patient relationship, embarrassment or the perceived irrelevance to the clinical situation. GDPs expressed low confidence in approaching alcohol related problems. Conclusions GDPs felt that alcohol based discussions in primary care would not be relevant and would inevitably lead to disruption of the patient-clinician relationship. Further research is necessary to more fully understand the attitudes, behaviour and knowledge of GDPs regarding the provision of alcohol related health advice. The results of this study have informed the design of a paper postal survey for wider distribution.

\section{INTRODUCTION}

Alcohol misuse has high social, economic and health costs. The trends towards increased affordability and availability of alcohol and the consequential harm is evident in ever-increasing alcohol-related crime, health service utilisation, and alcohol related morbidity and mortality. ${ }^{1,2}$ The cost of alcohol misuse to the Scottish economy constitutes a major financial burden, with estimates in excess of $£ 1$ billion annually.,4

Liver cirrhosis mortality rates have increased sharply in the United Kingdom, bluntly opposing the general downward trend in other European countries.

${ }^{1 *}$ Lecturer in Oral Surgery, Unit of Oral Surgery \& Medicine, University of Dundee Dental Hospital \& School, Park Place, Dundee, Scotland, DD1 4HN; ${ }^{2}$ Research and Development Manager, Scottish Dental Clinical Effectiveness Programme, Dundee Dental Education Centre; ${ }^{3}$ Director of the Effective Dental Practice Programme, Dental Health Services Research Unit; ${ }^{4}$ Senior Research Fellow, Dental Health Services Research Unit, University of Dundee; ${ }^{5}$ Professor of Oral Surgery, Unit of Oral Surgery \& Medicine, University of Dundee Dental Hospital \&t School

${ }^{*}$ Correspondence to: Dr Simon Shepherd

Email: sdshepherd@dundee.ac.uk

\section{Online article number E13}

Refereed Paper - accepted 24 November 2009

DOI: $10.1038 /$ sj.bdj.2010.342

${ }^{\circledR}$ British Dental Journal 2010; E13
Scotland has witnessed a six-fold increase in liver mortality rate since the $1950 \mathrm{~s}^{5}$ and has one of the highest cirrhosis mortality rates in western Europe. The most palpable cause is the increase in overall alcohol consumption. ${ }^{5}$

Early identification of alcohol related problems offers a gateway to affect change before the onset of the overt physical, psychological or social impediment associated with alcohol dependence. ${ }^{6}$

Primary care is often identified as the most appropriate place to undertake the early identification screening process and subsequently deliver a brief intervention (BI) aimed at reducing alcohol consumption. Indeed there are current guidelines suggesting that all health care professionals should be opportunistically screening patients for alcohol excess and offering advice, notably the Scottish Intercollegiate Guidelines Network - (SIGN) The Management of Harmful Drinking and Alcohol Dependence in Primary Care guideline.?

Several comprehensively validated questionnaire-based screening tools are available to elucidate risk patterns related to alcohol consumption..$^{8-10}$
BIs are motivational counselling type supportive discussions, which last as little as 5-15 minutes with the aim of promoting behaviour change. There is a large body of evidence highlighting the successful use of BIs, ${ }^{11,12}$ with some authors suggesting reduction in alcohol consumption for periods of six up to 48 months. ${ }^{13,14}$ Indeed a recent article proposed that the clinical relevance of alcohol BI is such that delaying dissemination will result in otherwise avoidable deaths..$^{15}$ It is generally agreed that BIs offer an efficacious and cost effective way of decreasing the burden from excessive alcohol consumption. ${ }^{16,17}$ However, there are some detractors to BI, questioning their true effectiveness and advocating that further research regarding the evaluation and implementation of BI in primary care settings is undertaken. ${ }^{11,12,18}$

Alcohol is an established and significant risk factor for cancers of the mouth, larynx, pharynx and oesophagus ${ }^{19}$ and this association may provide the unique link to bind together dentistry and the provision of alcohol related discussion during consultation in primary care.

The established evidence of a link between alcohol and oral cancer ${ }^{20}$ should 
provide the necessary motivation for a dentist who is primarily concerned with managing conditions of the oral cavity. Indeed the National Institute for Health and Clinical Excellence (NICE) recall guidelines ${ }^{21}$ advise that GDPs should discuss aetiological risk factors with patients.

The diagnosis of oral cancer in primary care is often said to be a rare and chance finding (and the link with alcohol consumption might not be immediately noted); nonetheless the potential significance and consequence for the patient is such that we would argue that it should be adopted as a preventive strategy by those primarily concerned with oral health.

Although approximately 57\% of adults are registered with a National Health Service dentist in Scotland ${ }^{22}$ it has been estimated that up to $80 \%$ of the population will access NHS dental services over a six year period..$^{23}$ In 2007-2008 NHS GDPs performed 2.2 million examinations ${ }^{22}$ in Scotland alone and it is this high contact exposure to the population that accentuates the potential for GDPs to play an integral role in both the detection and the prevention of oral cancer primarily through discussion of the major risk factors of alcohol misuse and tobacco smoking. ${ }^{24}$

However, it is already known that there are numerous barriers to implementing alcohol BIs in the primary health care setting by general medical practitioners. These barriers include negative clinician attitude, insufficient skills or knowledge, and inadequate resources or support. ${ }^{25}$ Little is known or understood about GDP attitudes, behaviours and beliefs regarding their potential role in screening for alcohol misuse in the primary dental care environment.

The aim of this exploratory study was to identify salient beliefs of GDPs relating to the identification of alcohol misuse and the provision of alcohol related health advice and to identify any barriers or facilitators to introducing this into general practice.

\section{METHOD}

Semi structured interviews were conducted with a convenience sample of GDPs in the North Highland region of Scotland. Each was invited to participate in an interview lasting approximately ten minutes. All approached consented to participate.
A total of 12 GDPs were interviewed with two being independent practitioners and ten salaried practitioners, of which three were female and nine were male with a wide range of both age and experience. Interviews were conducted by a single interviewer and an interview schedule employed to ensure coverage of subject matter during the interview process. Responses were transcribed directly on to the interview schedule during the interview process. Additional comments not directly related to the questions were incorporated as necessary.

An inductive approach was used with subsequent basic thematic content analysis performed to gather and order the responses into relevant categories based on the emerging themes.

\section{RESULTS}

The content analysis revealed four main themes regarding the role of the GDP in discussing alcohol related issues with patients:

- Recognition of the impact of alcohol and oral or general health

- Knowledge base regarding alcohol

- Current practices

- Views on providing alcohol advice (barriers/facilitators, advantages/ disadvantages, confidence).

All of the respondents believed that alcohol consumption does have an effect on general health, and all but one positively identified alcohol consumption as having an impact on oral health:

'Linked with smoking there is a strong correlation with oral cancer - spirits, smoking and oral cancer.'

'Linked with calories, people don't eat nutritional foods, and have deficiencies.'

Half of respondents reported knowing the Department of Health recommendations ${ }^{26}$ of 3-4 units per day for a man and 2-3 units per day for a woman with no more than 21 units per week for a man and 14 for a woman, yet subsequently reported them incorrectly; one third could recite them accurately, while two respondents admitted that they were not aware of any guidelines at all. All GDPs reported consuming alcohol.

Regarding knowledge of any guideline suggesting that alcohol should be discussed by the GDP during routine consultation three GDPs mentioned non-specific oral cancer guidelines, seven were unaware of any guideline and two reported awareness of guidelines issued from organisations such as the National Institute for Health \&t Clinical Excellence (NICE) and the British Dental Association (BDA).

'Yes, (my) own reading...it's just good advice.'

'Yes, the NICE recall guideline... Oral cancer.'

\section{'No... British Dental Association?'}

Nine GDPs reportedly recorded alcohol consumption. Five reported 'Yes' to recording alcohol consumption but advised that this was on the medical history questionnaire. Two reported 'No' to the same question but qualified the statement by adding that they had done so on the medical history questionnaire.

It was clear that seven GDPs were passively enquiring or recording via a surrogate mechanism, referring to the request for the patient to record units per week on the (non-specified) medical history questionnaire form, with no further active questioning.

One quarter did not ask, did not record and did not recount using a medical history questionnaire as an indicator of consumption. On asking patients about alcohol:

'No, but I know by just looking. Well, yes, the medical history questionnaire.'

Only one GDP discussed with patients their alcohol intake if it was found to be excessive, with eight never doing so irrespective of consumption and three doing it 'sometimes'.

Confidence was reportedly low when discussing alcohol. One GDP reported being confident in discussing difficult issues such as alcohol with patients. Five respondents were confident only sometimes or only if there was a clinical indication to do so, with the presence of oral lesions considered a potential motivator to enter alcohol related discussion. On confidence:

'No. [I am] not qualified to do so. [I am] confident with dental issues though.'

'Sometimes [I feel confident]. [I] Would do [feel confident] with better training, like addiction support workers, and knowing how to do it and deal with problems.'

'Not if there is no clinical indication to do so.'

The main barriers cited included disruption of the patient-clinician relationship 
(8/12), adverse patient behaviour (6/12), embarrassment (5/12), relevance (5/12) and causing offence (3/12). Only one GDP reported limited time as a barrier to providing alcohol related advice.

A grouping of the responses that might broadly be considered under the umbrella term of disruptive to the patient-clinician relationship (including mention of clinician-patient relationship, embarrassment, causing offence and adverse behaviour) indicated that $11 / 12$ GDPs would view potential relationship imbalance or interference a potent underlying barrier to asking about alcohol consumption.

'Patients feel they are being criticised, they get embarrassed, leading to antagonising behaviour and non-compliance with treatment plans.'

'There is an economic relationship ... a business. It promotes antagonistic behaviour. It is service specific, hospital dentists and salaried might afford to ask, but private practitioners ... there's an economic relationship.'

'Embarrassing patients. It would interfere with the relationship (dentist-patient). It is a privacy issue.'

'...it's disruptive of the dentist-patient relationship.'

Relevance, or the perceived lack of relevance to the practice of dentistry, was a purported barrier for five GDPs. Relevance issues were indicated for both the patient and dentist with GDPs assigning it as irrelevant and also presupposing that patients will consider it irrelevant.

'Patients don't see the relevance and they may not be honest. Might be more relevant to the community and salaried services.'

'It's not the patients' expectation of the role of a dentist; patients would not see the relevance.'

'It's not relevant... [I] don't ask.'

Nearly half (5/12) indicated that the relationship between oral cancer or the presence of oral lesions is an advantage offering a positive clinical indication to enter into alcohol related discussion and that this could improve health outcomes:

'Improving oral health, reducing oral cancer.'

'Yes, it could potentially influence health outcomes.'

'Yes. Potential lesions ... white patches ... keratoses ... [We] can then discuss the aetiology.'
Potential management options for those identifying at-risk drinking behaviour in attending patients were perceived to be limited. However, despite this perception four GDPs proposed that they might be able to offer advice, five might refer to the patient's own GMP and one was aware of, and willing to refer to, an unspecified telephone helpline service.

Two GDPs identified a lack of management pathway as an overt obstacle to questioning patients, arguing that if there is no pathway or mechanism available once the information is gained, then there is little point in undertaking the behaviour:

'Yes, but only if you will do something with the information, you know, further investigations...clotting/coag[ulation] screen.'

'Not really sure what to do with the information. There's no pathway other than giving the phone number.'

One third of respondents felt that there would be no advantage at all to questioning and discussing alcohol consumption:

'Added information won't change behaviour - it's too big a problem.'

'No influence.. negligible.'

The respondents perceived that patients would not appreciate the delivery of alcohol advice as a legitimate role for a GDP and that any measures taken to promote alcohol screening and the provision of advice, in essence endorsing the role legitimacy among patients, would be a persuasive facilitator. Five GDPs thought a media campaign directed at patients would facilitate the behaviour, five GDPs suggested training or education of dentists, while non-specific support was identified by four GDPs as a potential catalyst for undertaking the behaviour. It was clear that a wider awareness of the GDP's role in alcohol advice delivery was a perceived facilitator:

'Prior notification of the relevance... patient education.'

'A display or media campaign why it is relevant ... so patients know. A patient information leaflet ... or CD-ROM maybe.'

'Awareness. Awareness in the media for patients ... for dentists ... postgraduate courses.'

Remuneration was seen as a facilitator by one GDP, while four felt that nothing at all could be done to facilitate GDPs entering into alcohol related discussion.

\section{DISCUSSION}

This explorative study identified salient beliefs concerning the involvement of GDPs in alcohol screening and delivery of alcohol related health advice, with specific interest in the barriers and facilitators to such provision.

The present study illustrates that GDPs articulate in concert the association between alcohol and general health, with the majority also appreciating a similar association with oral health. This might be considered consistent with previous work suggesting that the majority (87\%) of GDPs indeed recognise alcohol as a risk factor for oral cancer. ${ }^{27}$ However, there has hitherto been a failure to translate this into wider discussion about alcohol consumption during dental consultation; indeed it has been found that only 3\% of GDPs routinely enquire about alcohol consumption..$^{28}$

Knowledge of recommended intake guidelines was, however, poor. Indeed the proportion of GDPs in this study who were able to accurately relay the recommended safe daily intake guidelines was less than that of the general population (Office for National Statistics, 2009). ${ }^{29}$ This might be an indicator of a basic training requirement.

It was also clear that GDPs have a peripheral awareness that alcohol questioning should constitute part of an overall patient assessment. GDPs emphasised the fact that the issue of alcohol had been raised and recorded in the medical history questionnaire, even if the patient was not directly questioned. In addition the provision of alcohol advice was mentioned under various umbrella organisations such as the BDA, NICE and non-specific 'oral cancer' guidelines.

The results suggest that GDPs think that the issue of alcohol intake should be recorded in the medical history questionnaire. However, their apparent reluctance to raise the alcohol issue with their patients also suggests that GDPs may lack the motivation, confidence or conviction that they can successfully do so.

Although superficially it might seem positive that the majority of GDPs recorded alcohol consumption, it was a passive and empty act. Often the recording came with no further discussion or advice regarding excessive consumption and in that regard is essentially a valueless exercise. 
This potentially raises an ethical quandary associated with GDPs collecting information for no explicit purpose. Failure to act on any information collected may have important and potentially significant adverse consequences for the patient. It may be perceived that the recording of information without subsequently engaging in an appropriate action or behaviour may constitute medico-legal negligence.

Only one GDP did discuss excessive alcohol intake with patients. Further research might seek to investigate the attitudes and beliefs of practitioners that do indulge in the behaviour and determine what differentiates them from those who do not. This may aid subsequent development of interventions designed to target and enhance these beliefs in GDPs, leading to increase likelihood they will undertake the behaviour.

A lack of confidence was experienced by GDPs preventing them entering into discussion about alcohol unless it was associated with a pivotal clinical event, such as the presence of oral lesions. Such confidence problems may be linked to inadequate training, perceived problems with relevance or a perception of poor efficacy in entering the discussion and ultimately reducing alcohol consumption. Any planned intervention to support GDPs might seek to redress this confidence problem by promoting belief in the importance of providing alcohol advice and that GDP involvement is not only valuable but also integral to patient management.

The primary cited barriers centre on disruption of the patient-clinician relationship. This might not seem unusual as the relationship acts as an important medium for the exchange of information and concerns, expressing feelings, and is fundamental to successful treatment and satisfaction of both patient and practitioner.

Financial concerns and economic upset might be postulated as possible disincentives to engaging in potentially disruptive discussions around alcohol excess. However, our sample consisted primarily of salaried GDPs, a group less likely affected by such financial complication, hence one might suggest that there are other more important values within the patient-clinician relationship that GDPs are loath to disrupt.
Maybe paradoxically GDPs, as health care professionals, felt that alcohol screening was not relevant, despite the near universal agreement that alcohol affects both oral and general health. Asking GDPs to question patients about what they view as an embarrassing, uncomfortable and potentially disruptive subject with no perceived relevance to the practice of dentistry is likely to be fruitless. However, this relevance issue was juxtaposed with the collective acceptance that oral cancer and alcohol are inextricably linked.

The results suggest that GDPs are aware of alcohol as a risk factor for oral cancer and this may offer a potential portal of entry for dental practitioners to introduce the concepts of harm and alcohol excess to the patient under the umbrella of oral disease and risk factor awareness, principally regarding oral cancer risk. In this guise it may be verbalised in the GDP's own sphere of professional comfort, and indeed professional confidence.

Limited management options are an overt obstacle to entering the behaviour, even at the source of recording consumption. GDPs are adept at information gathering and do it for both clinical and administrative purposes, but it generally does have a purpose. Clear management options are needed such that a patient, once identified with a potential problem, has a readily recognised route to follow in order to receive appropriate advice or care and that this pathway is practicable and fully supported.

The perceived main facilitators appear to surround education. The primary proposed facilitator aimed at patients was development of a media or awareness campaign to emphasise the dangers of alcohol and to ensure that they are forewarned to expect alcohol related questioning when attending primary care dental services. It is plausible that this type of advanced patient awareness would also simultaneously legitimise the role in advance for the GDP, automatically negating the perceived barrier of relevance.

For GDPs the combination of further training with the presence of a published guideline has been postulated as a facilitating influence. It is, however, known that education and traditional passive dissemination of guidelines alone are often not sufficient to automatically affect behaviour change $\mathrm{e}^{30,31}$ and that other cofactors are often required, especially support mechanisms.

\section{CONCLUSION}

The prospect of GDP involvement in alcohol related issues was received with negativity and scepticism. GDPs expect that it would result in unrest and disruption, despite evidence that the majority of patients are quite accepting of this type of questioning by a dental professional. ${ }^{32}$

GDPs universally accept the negative pathogenic role of alcohol on health but this understanding alone has not been sufficient to affect change. GDPs are not comfortable with alcohol related discussion in primary care and feel they lack confidence, support and appropriate management pathways.

This exploratory study has informed the design and content of a theoretically based postal questionnaire to further appreciate GDP views on alcohol and to understand GDP beliefs, motivation and attitudes towards providing alcohol related health advice including the concern over patientpractitioner relationships.

The ultimate goal is the development of an intervention to enable GDPs to better communicate alcohol related oral health risk to patients under the umbrella of oral cancer risk factor awareness.

1. The Substance Misuse Information Strategy Team. Alcohol Statistics Scotland 2009. Report. Edinburgh NHS National Service Scotland, National Statistics, Information Services Division, 2009.

2. Office for National Statistics. Health Statistics Quarterly, No 33 Spring: National Statistics; 2007. February 2007.

3. Scotland A. Drug and alcohol services in Scotland. Report Edinburgh: Audit Scotland 2009, March 2009.

4. Scottish Executive. Cost to Society of Alcohol Misuse in Scotland. An Update to 'Alcohol Misuse in Scotland Trends and Costs' (Scottish Executive, October 2001). Health Economics Unit ASD. Scottish Executive, 2004. 12 July 2004

5. Leon D A, McCambridge J. Liver cirrhosis mortality rates in Britain from 1950 to 2002: an analysis of routine data. Lancet 2006; 367: 52-56.

6. Cheeta S, Drummond C, Oyefeso A, Phillips T et al. Low identification of alcohol use disorders in general practice in England. Addiction 2008: 103: 766-773.

7. Scottish Intercollegiate Guidelines Network. The management of harmful drinking and alcohol dependence in primary care: a national clinical guideline. Edinburgh: Scottish Intercollegiate Guidelines Network, 2003.

8. Aertgeerts B, Buntinx F, Ansoms S, Fevery J. Screening properties of questionnaires and laboratory tests for the detection of alcohol abuse or dependence in a general practice population. BrJ Gen Pract 2001; 51: 206-217.

9. Bush K, Kivlahan D R, McDonell M B, Finn S D, Bradley K A. The AUDIT alcohol consumption questions (AUDIT-C): an effective brief screening 
test for problem drinking. Ambulatory Care Quality Improvement Project (ACQUIP). Alcohol Use

Disorders Identification Test. Arch Intern Med 1998; 158: 1789-1795.

10. Gual A, Segura L, Contel M, Heather N, Colom J. Audit-3 and audit-4: effectiveness of two short forms of the alcohol use disorders identification test. Alcohol Alcohol 2002; 37: 591-596.

11. Ballesteros J, Duffy J, Querejeta I, Arino J, GonzalezPinto A. Efficacy of brief interventions for hazardous drinkers in primary care: systematic review and meta-analyses. Alcohol Clin Exp Res 2004; 28: 608-618.

12. Kaner E F, Beyer F, Dickinson H O, Pienaar E et al. Effectiveness of brief alcohol interventions in primary care populations. Cochrane Database Syst Rev 2007 (2): CD004148.

13. Bertholet N, Daeppen J B, Wietlisbach V, Fleming $M$, Burnand B. Reduction of alcohol consumption by brief alcohol intervention in primary care - systematic review and meta-analysis. Arch Intern Med 2005; 165: 986-995.

14. Fleming M F, Mundt M P, French M T, Manwell L B et al. Brief physician advice for problem drinkers: long-term efficacy and benefit-cost analysis. Alcohol Clin Exp Res 2002; 26: 36-43.

15. Cuijpers $P$, Riper $H$, Lemmers $L$. The effects on mortality of brief interventions for problem drinking: a meta-analysis. Addiction 2004; 99: 839-845.

16. Kaner E F, Heather N, McAvoy B R, Lock C A, Gilvarry E. Intervention for excessive alcohol consumption in primary health care: attitudes and practices of English general practitioners. Alcohol Alcohol 1999;
34: 559-566.

17. Kaner E, Rapley T, May C. Seeing through the glass darkly? A qualitative exploration of GPs' drinking and their alcohol intervention practices. Fam Pract 2006; 23: 481-487.

18. Richmond R, Heather N, Wodak A, Kehoe L, Webster I. Controlled evaluation of a general practice-based brief intervention for excessive drinking. Addiction 1995; 90: 119-132.

19. Martin-Moreno J M, Soerjomataram I, Magnusson G. Cancer causes and prevention: a condensed appraisal in Europe in 2008. Eur J Cancer 2008; 44: 1390-1403.

20. Bagnardi V, Blangiardo M, La Vecchia C, Corrao G. Alcohol consumption and the risk of cancer: a meta-analysis. Alcohol Res Health 2001 25: 263-270.

21. National Institute for Clinical Excellence. Dental recall: recall interval between routine denta examinations. Clinical Guideline 19. London National Institute for Clinical Excellence, 2004 October 2004.

22. ISD publications. Dental examinations in Scotland, year ending March 2008. Edinburgh: NHS National Services Scotland. Information Services Division. Scotland, 2008. 31 March 2008. http://www. isdscotland.org/isd/4684.html

23. Tilley C J, Chalkley M J. Measuring access to health services: general dental services in Scotland. Br Dent J 2005; 199: 599-601, discussion 583.

24. MacPherson L M, McCann M F, Gibson J. The role of the general dental practitioner in detection and prevention of oral cancer: a review of the literature.
Dent Update 2000; 27: 404-408.

25. Nilsen $P$, Aalto M, Bendtsen P, Seppa K. Effectiveness of strategies to implement brief alcohol intervention in primary healthcare - a systematic review. Scand J Prim Health Care 2006; 24: 5-15.

26. Department of Health. Alcohol Advice. [Online]. 2009 [cited 23 April 2009]. Available from: http://www.dh.gov.uk/en/Publichealth/ Healthimprovement/Alcoholmisuse/DH_085385.

27. Carter L M, Ogden G. Oral cancer awareness of general medical and general dental practitioners. Br Dent J 2007; 203: E10, discussion 248-249.

28. Macpherson L M, McCann M F, Gibson J, Binnie V I, Stephen $\mathrm{K} \mathrm{W}$. The role of primary healthcare professionals in oral cancer prevention and detection. Br Dent J 2003; 195: 277-281, discussion 63.

29. Lader D. Drinking: adults' behaviour and knowledge in 2008. Cardiff: The Office for National Statistics, NHS Information Centre, 2009.

30. McGlone P Watt R, Sheiham A. Evidence-based dentistry: an overview of the challenges in changing professional practice. Br Dent J 2001; 190: 636-639.

31. Eccles M, Grimshaw J, Walker A, Johnston M, Pitts $\mathrm{N}$. Changing the behavior of healthcare professionals: the use of theory in promoting the uptake of research findings. J Clin Epidemio/ 2005; 58: 107-112.

32. Miller P M, Ravenel M C, Shealy A E, Thomas S. Alcohol screening in dental patients: the prevalence of hazardous drinking and patients' attitudes about screening and advice. J Am Dent Assoc 2006; 137: 1692-1698, quiz 730-731. 